\title{
Distribution of heterochromatin and rDNA on the holocentric chromosomes of the aphids Dysaphis plantaginea and Melanaphis pyraria (Hemiptera: Aphididae)
}

\author{
Angela CRINITI ${ }^{1}$, Gabriele SimONAZZI ${ }^{1}$, Stefano CASSANELLI ${ }^{1}$, Mario FERRARI ${ }^{1}$, \\ DAVIDE BIZZARO ${ }^{2}$ and Gian CARLO MANICARDI ${ }^{1 *}$ \\ ${ }^{1}$ Dipartimento di Scienze Agrarie e degli Alimenti, Università di Modena e Reggio Emilia, Via Amendola 2, 42100 Reggio Emilia, \\ Italy; e-mail: giancarlo.manicardi@unimore.it \\ ${ }^{2}$ Dipartimento di Biochimica, Biologia e Genetica, Università Politecnica delle Marche, Ancona, Italia
}

Key words. Aphid, Cytogenetics, FISH, Holocentric chromosomes, Heterochromatin, rDNA size heteromorphism

\begin{abstract}
The structure of the holocentric chromosomes of the rosy apple aphid, Dysaphis plantaginea $(2 \mathrm{n}=12)$, and pear-grass aphid, Melanaphis pyraria $(2 \mathrm{n}=8)$, was studied using C-banding, NOR, Giemsa and fluorochrome staining, and fluorescent in situ hybridization (FISH). Contrary to the equilocal distribution of heterochromatin typical of monocentric chromosomes, in both species C-banding evidenced a tendency of highly repetitive DNAs to be restricted to the X chromosomes. Silver staining and FISH, using a 28S rDNA probe, located rDNA genes on one telomere of each X chromosome, the only brightly fluorescent C-positive sites revealed by $\mathrm{CMA}_{3}$ staining, whereas all other heterochromatic C-bands were DAPI positive. Both species showed a noticeable amount of rDNA heteromorphism. Mitotic recombination is proposed as a possible mechanism responsible for the variation in size of rDNA.
\end{abstract}

\section{INTRODUCTION}

Most eukaryotes possess monocentric chromosomes characterised by a single localized centromere, which, under a microscope, appears as a primary constriction in the chromosome. At anaphase, in species with monocentric chromosomes, microtubules attach to the centromeres and bring the chromosomes to the pole, with the centromere leading. By contrast, holocentric chromosomes lack a distinct primary constriction and centromeric activity is spread along the entire length of the chromosomes. During mitosis, microtubules become attached to almost the entire length of each chromosome and as a result they each move in the form of a straight rod towards a pole (Hughes-Schrader \& Schrader, 1961). However, during meiosis kinetic activity is restricted to the telomeric regions and the chromosomes can be regarded as telokinetic (Nokkala, 1985).

Compared with many species with monocentric chromosomes, very few have holocentric chromosomes. Nevertheless, species with holocentric chromosomes are recorded in both the plant and animal kingdoms, including insects and nematodes, which is thought to be a result of convergent evolution (Dernburg, 2001). Notwithstanding their uniqueness, holocentric chromosomes have not been intensively studied, except in the nematode worm Caenorhabditis elegans (for a review see Maddox et al., 2004),

Mitotic chromosomes can be easily observed in aphid embryonic tissues. For this reason, aphids represent a suitable insect model for determining the differences and similarities in the structure and activity of holocentric and monocentric chromosomes. The identification of chromosomal markers in organisms possessing holocentric chromosomes is hampered by the lack of a simple morphological index (i.e. a centromere) and of clear-cut banding patterns (Blackman, 1987; Hales et al., 1997; Manicardi et al., 2002). Indeed, the development of cytogenetic tools in aphid species is becoming crucial for mapping and positional cloning of orthologous genes coming from the genome project of the pea aphid Acyrthosiphon pisum which is currently under the annotation step (www.hgsc.bcm.tmc.edu/projects/aphid/). These resources will provide novel opportunities for investigating many peculiar features of aphid biology, such as aphid-plant interactions and insecticide resistance in aphid species of agricultural importance.

The present paper reports the results of a cytogenetic study of two pests of pome orchards: the rosy apple aphid, Dysaphis plantaginea (Passerini), which is an important pest in western European apple orchards and particularly troublesome in organic apple production (Cross et al., 2006), and the brown pear aphid, Melanaphis pyraria, which in addition to causing direct damage also transmits barley yellow dwarf luteovirus (El Yamani \& Hill, 1991).

\section{MATERIAL AND METHODS}

Aphids used in this study originated from field populations near Reggio Emilia (Northern Italy). Specimens of Dysaphis plantaginea were collected from apple trees and Melanaphis pyraria from pear orchards.

\footnotetext{
* Corresponding author.
} 

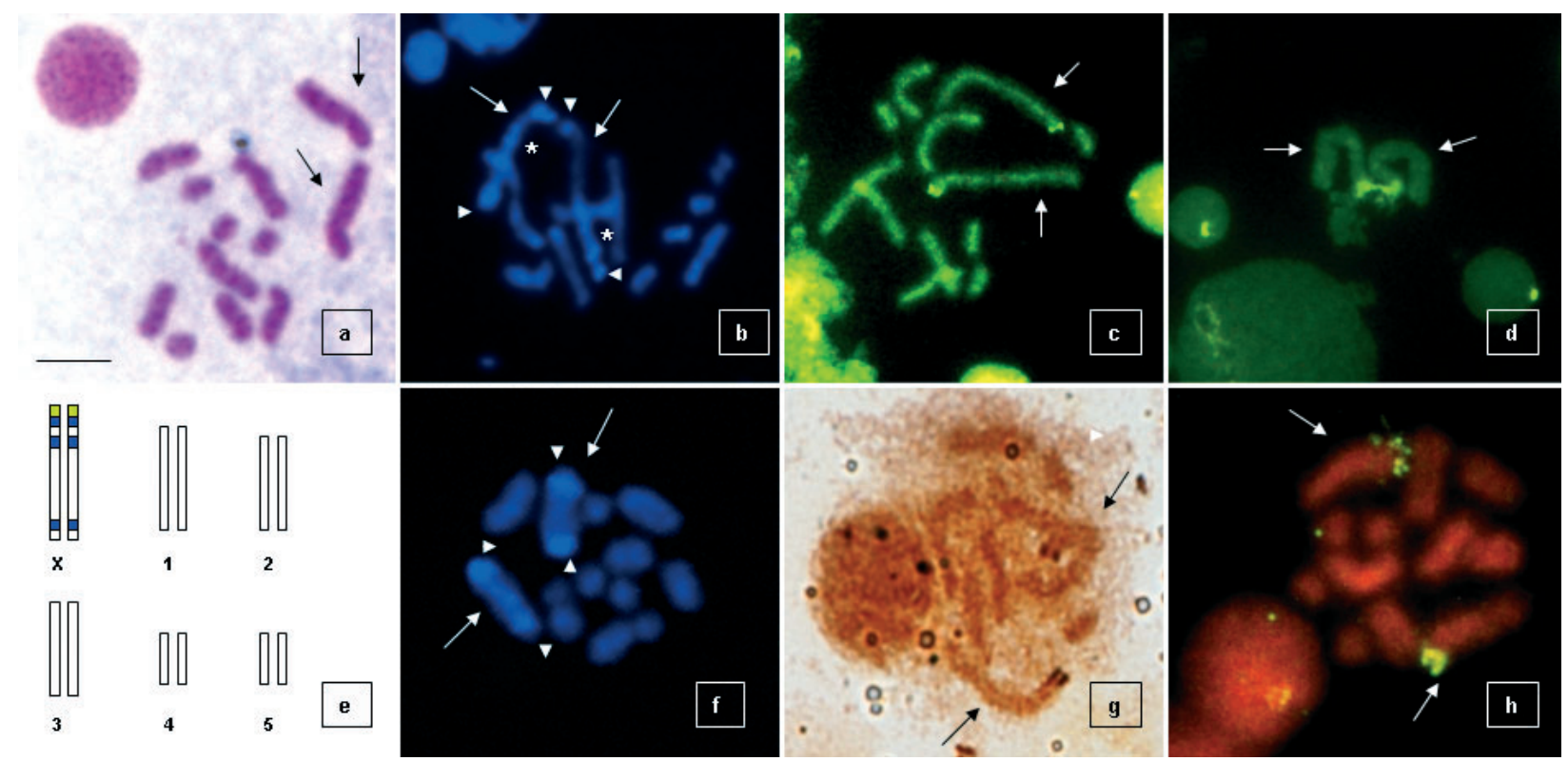

Fig. 1. Mitotic chromosome complements of Dysaphis plantaginea at metaphase after Giemsa staining (a); late prophase after DAPI-staining (b) and metaphase (f) after C-banding; late prophase after $\mathrm{CMA}_{3}$ staining (c, d); metaphase after silver staining (g); metaphase after FISH with the 28S rDNA probe (h). The ideogram (e) summarises the C-banding pattern in the D. plantaginea karyotype, AT-rich (blue) and GC-rich (green) chromatin. Arrows indicate X chromosomes; arrowheads and asterisks indicate telomeric and intercalary C-bands, respectively. Bar $=10 \mu \mathrm{m}$.

Chromosome spreads of embryonic cells obtained from hundreds of parthenogenetic females were prepared as previously described by Manicardi et al. (1996). C-banding treatment was performed according to the procedure described in Sumner (1972). After this treatment, some slides were stained with 5\% Giemsa solution in Sörensen buffer, pH 6.8, for 10 min. Chromomycine $\mathrm{A}_{3}\left(\mathrm{CMA}_{3}\right)$ staining was carried out as described in Schweizer (1976) and the 4'-6'-diamidino-2-phenylindole (DAPI) treatment as described by Donlon \& Magenis (1983). Silver staining of nucleolar organizing regions (NORs) was performed following the technique of Howell \& Black (1980).

DNA extraction from aphid embryos was carried out as described in Bizzaro et al. (1996). Two species specific 28S rDNA probes were obtained by PCR amplification of $D$ plantaginea and $M$. pyraria genomic DNAs, respectively, using two primers, forward (5'-AACAAACAACCGATACGTTCCG-3') and reverse (5'-CTCTGTCCGTTTACAACCGAGC-3'), designed according to the coding $28 \mathrm{~S}$ sequence of the aphid Acyrthosiphon pisum (GenBank acc. no. X66419) (Amako et al., 1996). The amplification mix contained $100 \mathrm{ng}$ genomic DNA, $1 \mathrm{mM}$ of each primer, $200 \mathrm{mM}$ dNTPs and $2 \mathrm{U}$ of DyNAZyme II DNA polymerase (Finnzymes Oy, Espoo, Finland). The amplification was performed with a thermocycler Hybaid (Hybaid Ltd, Basingstoke, UK). PCR amplification conditions were $2 \mathrm{~min}$ of denaturation at $94^{\circ} \mathrm{C}$, followed by 30 cycles of $94^{\circ} \mathrm{C}$ for $30 \mathrm{~s}, 60^{\circ} \mathrm{C}$ for $1 \mathrm{~min}$, and $72^{\circ} \mathrm{C}$ for $1 \mathrm{~min}$, with final extension step of $72^{\circ} \mathrm{C}$ for $10 \mathrm{~min}$. Probe labeling and fluorescent in situ hybridization (FISH) were performed according to Bizzaro et al. (1996).

\section{RESULTS}

Dysaphis plantaginea metaphases, observed in embryonic cells, revealed a chromosome number of $2 \mathrm{n}=12$ (Fig. 1a). Melanaphis pyraria metaphases revealed a chromosome number of $2 \mathrm{n}=8$ (Fig. $2 \mathrm{a}$ ).
DAPI staining of C-banded chromosomes of Dysaphis plantaginea revealed two telomeric and two faint intercalary bands on the X chromosomes (Fig. 1b). C-banding treatment in Melanaphis pyraria revealed one main heterochromatic band located in a telomeric position on the $\mathrm{X}$ chromosomes. The same chromosomes also have a number of slim intercalary bands, which are not always distinguishable (Fig. 2b). In both species, the telomeric $\mathrm{C}$-positive bands are always visible, independent of the degree of $\mathrm{X}$ chromosome condensation, whereas, in the more condensed metaphases intercalary bands are indistinguishable (Figs $1 \mathrm{f}$ and 2f). Both in D. plantaginea and $M$. pyraria, autosomes lack any kind of longitudinal differentiation (Figs $1 b$, f and $2 b-f$ ).

$\mathrm{CMA}_{3}$ staining of $\mathrm{C}$-banded mitotic metaphases in $D$. plantaginea and $M$. pyraria revealed a GC rich DNA region located at one telomere on each $\mathrm{X}$ chromosome (Figs $1 \mathrm{c}$ and $2 \mathrm{c}-\mathrm{f}$ ). In both species, the ends of the $\mathrm{X}$ chromosome that were brightly labelled with $\mathrm{CMA}_{3}$ fluorescence also stained with $\mathrm{AgNO}_{3}$, indicating remnants of argentophilic proteins (Figs $1 \mathrm{~g}$ and $2 \mathrm{~g}$ ). After FISH, using the $28 \mathrm{~S}$ rDNA probes, there were strong hybridization signals at the ends of the same X-chromosome (Figs $1 \mathrm{~h}$ and $2 \mathrm{~h}$ ). This suggests that in both $D$. plantaginea and $M$. pyraria this is the nucleolar organizing region (NOR), containing actively transcribed rDNA genes. In both species, we observed a substantial amount of heteromorphism between homologous rDNA sites after $\mathrm{CMA}_{3}$ and FISH using a rDNA probe (Figs $1 \mathrm{c}, \mathrm{h}$ and $2 \mathrm{c}, \mathrm{h}$ ). In some mitotic plates the two $\mathrm{X}$ chromosome were connected by their NOR bearing telomeres (Figs 1d and 2d). 

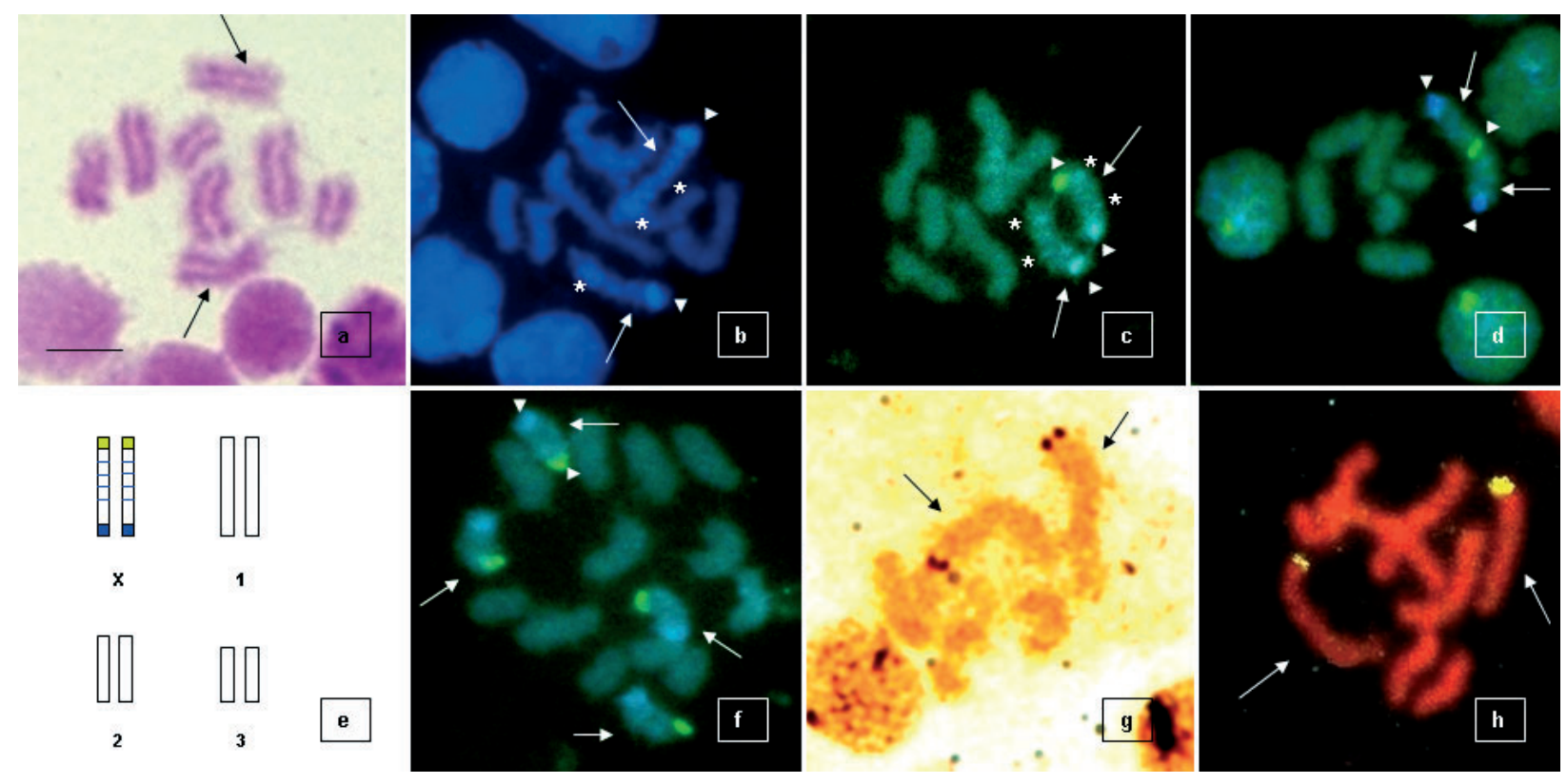

Fig. 2. Mitotic metaphase chromosomes of Melanaphys pyraria after Giemsa staining (a); DAPI staining (b) and combined DAPI$\mathrm{CMA}_{3}$ staining (c, d, f) after C-banding; silver staining (g); and FISH with the 28S rDNA probe (h). The ideogram (e) summarises the C-banding pattern in the M. pyraria karyotype, AT-rich (blue) and GC-rich (green) chromatin. Arrows indicate X chromosomes; arrowheads and asterisks indicate telomeric and intercalary C-bands, respectively. Bar $=10 \mu \mathrm{m}$.

\section{DISCUSSION}

In spite of the holocentric nature of aphid chromosomes, a high variation in chromosome number is recorded within the genus Amphorophora (Blackman, 1980) and Trama (Blackman et al., 2000), whereas most aphid genera have a remarkably stable number of chromosomes. For example, all of the Dysaphis species so far examined cytologically have $2 \mathrm{n}=12$. When chromosomal rearrangements occur, particularly fragmentations and translocations, they most commonly affect the autosomes, whereas the Xs, with few exceptions (Blackman, 1986; Hales, 1989), are the two longest chromosomes. The analysis of mitotic metaphases revealed diploid chromosome numbers for Dysaphis plantaginea $(2 \mathrm{n}=12)$ and Melanaphis pyraria $(2 \mathrm{n}=8)$ that accord with those previously recorded in the literature (Blackman \& Eastop, 2000)

From a cytogenetic point of view, the most important result is that heterochromatic bands were only found on the $\mathrm{X}$ chromosomes in these two species. However, it must be stressed that C-banding highlights only large blocks of highly repetitive DNA and, therefore, the presence of middle repetitive DNA at other sites of the chromosome complement can not be excluded. A different localization of C-positive heterochromatin on non homologous chromosomes of the same genome, which clearly contradicts "the equilocal distribution of heterochromatin" typical of monocentric chromosomes (Schweizer \& Loidl, 1987), is reported in other aphid species (Manicardi et al., 2002) but an exclusive localization on the $\mathrm{X}$ chromosomes has so far only been reported for two species of the Aphis genus, Aphis sambuci and A. pomi (Manicardi et al., 1998; Criniti et al., 2005). It has not always been possible to localize the heterochromatin in other organisms with holocentric chromosomes (Collet \& Westerman, 1984) but when possible, C-positive bands occur generally in telomeric and sometimes intercalary positions on the whole chromosome complement, (Camacho et al., 1985; Papeschi, 1988; Grozeva \& Nokkala, 2003; Guerra \& García, 2004). This suggests that the preferential, and in some case the exclusive localization of heterochromatin on $\mathrm{X}$ chromosomes is not a consequence of the holocentric structure but must be considered a peculiar feature of the aphid genome. Since heterochromatic blocks may influence the order of separation of sister chromatids in different chromosomes at anaphase (Vig, 1987), we hypothesize that the heterochromatic blocks restricted to $\mathrm{X}$ chromosomes are involved in the delay of $\mathrm{X}$ chromosome separation occurring during the maturation of the parthenogenetic oocyte, which is responsible for male sex determination in aphids (Blackman, 1987).

Sites of ribosomal rDNA genes (rDNA) can be identified by $\mathrm{Ag}$ staining, which visualize active NORs, by fluorescent staining with the antibiotic $\mathrm{CMA}_{3}$, which binds preferentially with GC-rich chromatin segments characteristically present at rDNA sites in aphids (Manicardi et al., 2002) and/or by in situ hybridization with rDNA probes. The constrained localization of rDNA cistrons on only one $\mathrm{X}$ telomere seems to be a general feature of aphids, since few exceptions are reported (Blackman \& Spence, 1996). This specific location could be the result of a strong evolutionary constraint related to the peculiar X0 male determination, which involves the pairing of the $\mathrm{X}$ chromosomes by their NORs during prophase of the maturation division (Blackman \& Spence, 1996). In D. plantaginea and M. pyraria, both $\mathrm{CMA}_{3}$ and FISH using $28 \mathrm{~S}$ rDNA probes revealed a noticeable 
variation in the size of rDNA on homologous X chromosomes. This finding strongly suggests that changes in intrachromosomal structure, involving rDNA loci, are responsible for the observed heteromorphism.

Variation in ribosomal gene copy number between homologous chromosomes has been attributed to various mechanisms, including unequal crossing over, transposition, tandem amplification and other rearrangements involving homologous segments, yielding structural modification of NORs (Castro et al., 2001). Connections between $\mathrm{X}$ chromosomes via their NOR-bearing telomeres were repeatedly observed in mitotic plates (Figs 1d and $2 \mathrm{~d}$ ), which suggests that unequal recombination at mitosis could be involved in the NOR heteromorphism observed in the aphids studied.

Recombination in mitotic cells is reported for several species, ranging from placental mammals (Cornforth \& Eberle, 2001; Svetlova et al., 2001) to insects (Stern, 1936; Bartsch et al., 1997) and yeast (Huang \& Keil, 1995). For example, in the yeast Saccharomyces cerevisiae, the recombination hotspot HOT1 initiates mitotic recombination when inserted into novel locations throughout the genome (Huang \& Keil, 1995). Among aphids, the occurrence of unequal mitotic recombination seems to be the more likely explanation for the intraindividual NORs heteromorphism reported in a parthenogenetic lineage of Megoura viciae (Mandrioli et al., 1999).

However, the use of polymorphic microsatellite markers did not reveal recombinant genotypes in parthenogenetically produced males or females (Hales et al., 2002), thus suggesting that either recombination or nonequal exchange between homologues is a peculiarity of rDNA sites rather than a general process affecting the whole X chromosome.

Further research is required to answer the intriguing question on the presence/benefit of mitotic recombination in aphids, whose reproductive strategy is largely characterized by apomictic parthenogenesis, which reduces (holocyclic strains) and in some cases excludes (obligate parthenogenetic strains) the possibility of genetic recombination by meiosis.

ACKNOWLEDGEMETS. This paper is dedicated to G. Simonazzi who died as a consequence of a road accident during the preparation of this manuscript. Dupont de Nemours partially supported this work.

\section{REFERENCES}

Аmako D., Kwon O.Y. \& Ishikawa H. 1996: Nucleotide sequence and presumed secondary structure of the $28 \mathrm{~S}$ rRNA of pea aphid: implication for diversification of insect rRNA. J. Mol. Evol. 43: 469-475.

Bartsch S., Würgler F.E. \& Sengstag C. 1997: A genetic system to detect mitotic recombination between repeated chromosomal sequences in Drosophila Schneider line 2 cells. Mutation Res./Genet. Toxicol. Environ. Mutagen. 395: 9-27.

Bizzaro D., Manicardi G.C. \& Bianchi U. 1996: Chromosomal localization of a highly repeated EcoRI DNA fragment in Megoura viciae (Homoptera, Aphididae) by nick translation and fluorescence in situ hybridization. Chromos. Res. 4: 392-396.
Blackman R.L. 1980: Chromosome numbers in the Aphididae and their taxonomic significance. Syst. Entomol. 5: 7-25.

Blackman R.L. 1986: The chromosomes of Japanese Aphididae (Homoptera), with notes on the cytological work of Orihay Shinji. Cytologia 51: 59-83.

BLACKMAN R.L. 1987: Reproduction, cytogenetics and development. In Minsk A.K. \& Harrewijn P. (eds): Aphids: Their Biology, Natural Enemies and Control. Vol. A. Elsevier, Amsterdam, pp. 163-195.

Blackman R.L. \& Eastop V.F. 2000: Aphids on the World's Crops: An Identification and Information Guide. Chichester, John Wiley \& Sons, 466 pp.

Blackman R.L. \& Spence J.M. 1996: Ribosomal DNA is frequently concentrated on only one $\mathrm{X}$ chromosome in permanently apomiptic aphids, but this does not inhibit male determination. Chromos. Res. 4: 324-320.

Blackman R.L., Spence J.M. \& Normark B.B. 2000: High diversity of structurally heterozygous karyotypes and rDNA arrays in parthenogenetic aphids of the genus Trama (Aphididae: Lachninae). Heredity 84: 254-260.

Camacho J.P.M., Belda J. \& Cabrero J. 1985: Meiotic behaviour of the holocentric chromosomes of Nezara viridula (Insecta, Heteroptera) analyzed by C-banding and silver impregnation. Can. J. Genet. Cytol. 27: 490-497.

Castro J., Rodríguez S., Pardo B.G., Sánchez L. \& Martínez P. 2001: Population analysis of an unusual NOR-site polymorphism in brown trout (Salmo trutta L.) Heredity 86: 291-302.

Collet C. \& Westerman M. 1984: Interspersed distribution patterns of C-bands and satellite DNA in the holocentric chromosomes of Luzula flaccida (Juncaceae). Genetica 63: 175-179.

CORNForth M.N. \& EBerLe R.L. 2001: Termini of human chromosomes display elevated rates of mitotic recombination. Mutagenesis 16: 85-89.

Criniti A., Simonazzi G., Cassanelli S., Ferrari M., Bizzaro D. \& MANICARDI G.C. 2005: X-linked heterochromatin distribution in the holocentric chromosomes of the green apple aphid Aphis pomi. Genetica 124: 93-98.

Cross J.W., Cubison S., Harris A. \& Harrington R. 2006: Autumn control of rosy apple aphid, Dysaphis plantaginea (Passerini), with aphicides. Crop Prot. 26: 1140-1149.

Danlon T.A. \& MAgENIS R.E. 1983: Methyl green is a substitute for distamycin $\mathrm{A}$ in the formation of distamycin A/DAPI C-bands. Hum. Genet. 65: 144-146.

DeRnBURG A.F. 2001: Here, there, and everywhere. Kinetochore function on holocentric chromosomes. J. Cell. Biol. 153: 33-38.

El Yamani M. \& Hill J.H. 1991: Aphid vectors of barley yellow dwarf virus in west-central Morocco. J. Phytopathol. 133: $105-111$.

Grozeva S. \& NokKala S. 2003: C-heterochromatin and extra (B) chromosome distribution in six species of the Nabis (Heteroptera, Nabidae) with the modal male karyotype 2n=/16XY. Folia Biol. (Kraków) 51: 13-22.

Guerra M. \& García M.A. 2004: Heterochromatin and rDNA sites distribution in the holocentric chromosomes of Cuscuta approximata Bab. (Convolvulaceae). Genome 47: 134-140.

HALES D.F. 1989: The chromosomes of Schoutedenia lutea (Homoptera, Aphididae, Greenideinae), with an account of meiosis in the male. Chromosoma 98: 295-300.

Hales D.F., Tomiuk J., Wohrmann K. \& Sunnucks P. 1997: Evolutionary and genetic aspects of aphid biology: A review. Eur. J. Entomol. 94: 1-55.

Hales D.F., Wilson A.C.C., Sloane M.A., Simon J.C., Le Gallic J.F. \& SunNucks P. 2002: Lack of detectable genetic recombination on the $\mathrm{X}$ chromosome during the parthenoge- 
netic production of female and male aphids. Genet. Res. 79: 203-209.

Howell W.M. \& BLACK D.A. 1980: Controlled silver-staining of nucleolus organizer regions with a protective colloidal developer: a 1-step method. Experientia 36: 1014-1015.

Huang G.S. \& KeIL R.L. 1995: Requirements for activity of the yeast mitotic recombination hotspot HOT1: RNA polymerase I and multiple cis-acting sequences. Genetics 141: 845-855.

Hughes-Schrader S. \& Schrader F. 1961: The kinetocore of the Hemiptera. Chromosoma 12: 327-350.

Maddox P.S., Oegema K., Desai A. \& Cheeseman I.M. 2004: "Holo"er than thou: chromosome segregation and kinetochore function in C. elegans. Chromosome Res. 12: 641-653.

Mandrioli M., Manicardi G.C., Bizzaro D. \& Bianchi U. 1999: NOR heteromorphism within a parthenogenetic lineage of the aphid Megoura viciae. Chromosome Res. 7: 157-162.

Manicardi G.C., Bizzaro D., Galli E. \& Bianchi U. 1996: Heterochromatin Heterogeneity in the holocentric X chromatin of Megoura vicine (Homoptera, Aphididae). Genome 39: 465-470.

Manicardi G.C., Mandrioli M., Bizzarro D. \& Bianchi U. 1998: Silver staining as a new bandin tecnique to identify aphid chromosome. Chromos. Res. 6: 55-57.

Manicardi G.C., Mandrioli M., Bizzarro D. \& Bianchi U. 2002: Cytogenetic and molecular analysis of heterochromatic areas in the holocentric chromosomes of different aphid spe- cies. In Sobti R.C., Obe G. \& Athwal R.S. (eds): Some Aspects of Chromosome Structure and Functions. Narosa Publishing House, New Delhi, pp. 47-56.

NoKKaLA S. 1985: Restriction of kinetic activity of holokinetic chromosomes in meiotic cells and its structural basis. Hereditas 102: 85-88.

PAPESCHI A.G. 1988: C-banding and DNA content in three species of Belostoma (Heteroptera) with large differences in chromosome size and number. Genetica 76: 43-51.

SCHWEIZER D. 1976: Reverse fluorescent chromosome banding with chromomycin and DAPI. Chromosoma 58: 307-324.

SchweIzer D. \& LoIDL J. 1987: A model for heterochromatin dispersion and the evolution of C-band patterns. Chromos. Today 9: 61-74.

STERN C. 1936: Somatic crossing-over and segregation in Drosophila melanogaster. Genetics 21: 625-730.

SUMNER A.T. 1972: A simple technique for demonstrating centromeric heterochromatin. Exp. Cell Res. 75: 304-306.

Svetlova E.Y., Razin S.V. \& Debatisse M. 2001: Mammalian recombination hot spot in a DNA loop anchorage region: a model for the study of common fragile sites. J. Cell Biochem. 36: $170-178$.

VIG B.K. 1987: Sequence of centromere separation. A possible role of repetitive DNA. Mutagenesis 2: 155-159.

Received December 5, 2008; revised and accepted January 16, 2009 